\title{
An Approach to Thermodynamics of Binary Substitutional Solutions
}

\author{
By Madan Lal Kapoor*
}

\begin{abstract}
A new approach, based on free volume theory, is developed for the calculation of thermodynamic functions of molten binary substitutional solutions. The calculations based on derived equations, which express the thermodynamic properties in terms of experimentally determinable variables at infinite dilution, show excellent agreement with experimental data on a large number of systems and are capable of quantitatively explaining the assymmetric behaviour of these systems. The theoretical variables are interpreted qualitatively in terms of fundamental properties of atoms.
\end{abstract}

(Received February 20, 1978)

\section{Introduction}

In recent years a number of models ${ }^{(1) \sim(6)}$ have been proposed to explain the thermodynamic properties of metallic solutions. These models which are based on the lattice assumptions have been comprehensively reviewed by Kapoor. ${ }^{(7)}$ The major weakness of these lattice models is that they do not take into account the free volume changes which occur on mixing. Thus, they are able to explain only approximately the experimental thermodynamic data.

In the present work a model based on free volume theory ${ }^{(8)}$ is proposed. The basic assumptions of this model are:

(i) A liquid metallic solution of substitutional components can be assumed to form a quasi-crystalline lattice with each atom having an average coordination number $Z$. This assumption is the same as the one made in the case of lattice models.

(ii) Each atom moves around its lattice site within a cell which is determined by the interaction of this atom with its nearest neighbours.

(iii) The energy of an atom is a function of the type of atoms in its first coordination shell. On the basis of these assumptions expressions for the free energy, enthalpy and entropy of mixing shall be derived in the next section.

* Department of Metallurgical Engineering, University of Roorkee, Roorkee-247672, India.

\section{Theoretical Treatment}

Consider a binary substitutional solution of $n_{A}$ atoms of component $A$ and $n_{B}$ atoms of component $B$. According to the free volume theory ${ }^{(8)}$, the configurational partition function of this system shall be given by the expression

$$
\begin{aligned}
\Omega_{c}= & \sum \prod_{\text {All }} \prod_{A \text { atoms }}\left(v_{A i}\right) \prod_{\text {All }}\left(v_{B j}\right) \\
& \times \exp \left[\left(-\frac{W_{0}}{k T}\right)\right],
\end{aligned}
$$

where summation in the above expression extends over all possible configurations of the system, $\Omega_{C}$ denotes the configurational partition function, $k$ and $T$ are Boltzmann's constant and absolute temperature respectively, $W_{0}$ is the energy of the system when all atoms are fixed on their lattice sites, $v_{A i}$ and $v_{B j}$ are the free volumes of $A$ and $B$ atoms having $i$ and $j$ atoms of components $B$ and $A$ respectively in their first coordination shells.

As the energy of the system is taken to be equal to the sum of the energies of all the atoms of the system, one can write for $W_{0}$ the expression

$$
W_{0}=\sum_{i=0}^{Z} n_{A i} W_{0 A i}+\sum_{j=0}^{Z} n_{B j} W_{O B j},
$$

where $n_{A i}$ and $n_{B j}$ are the numbers of $A$ and $B$ atoms respectively having $i B$ and $j A$ atoms in their first coordination shells, $W_{0 A i}$ and $W_{0 B j}$ are the energies of these atoms when they are fixed at their lattice sites.

1978 Vol. 19 
Writing together similar terms in the series of eq. (1), one gets the expression

$$
\begin{aligned}
\Omega=\frac{\Omega_{c}}{n_{A} ! n_{B} !}= & \sum g\left(n_{A}, n_{B}, n_{A i}, n_{B j}\right)\left[\exp \left\{\left(\frac{\sum_{i=0}^{\mathrm{Z}} n_{A i} W_{0 A i}+\sum_{j=0}^{Z} n_{B j} W_{0 B j}}{k T}\right)\right\}\right] \\
& \times \prod_{i=0}^{\mathrm{Z}}\left(v_{A i}\right)^{n_{A i}} \cdot \prod_{j=0}^{\mathrm{Z}}\left(v_{B j}\right)^{n_{B j}},
\end{aligned}
$$

where the summation now extends over all the different values of energy of the system, $g\left(n_{A}, n_{B}, n_{A i}, n_{B j}\right)$ is the number of different configurations corresponding to a given total energy value of the system.

The free energy of mixing of the system shall be given by

$$
\left(n_{A}+n_{B}\right) \Delta G_{\text {mix }}=-k T \ln \Omega-n_{A} W_{A 0}-n_{B} W_{B 0}-k T\left(n_{A} \ln v_{A 0}+n_{B} \ln v_{B 0}\right) .
$$

According to Mathieu et al. ${ }^{(9)(10)}, g\left(n_{A}, n_{B}, n_{A i}, n_{B j}\right)$ is given by

$$
g\left(n_{A}, n_{B}, n_{A i}, n_{B j}\right)=\frac{\left(n_{A}+n_{B}\right) !}{n_{A} ! n_{B} !} \cdot \frac{\prod_{i}\left(n_{A i}^{*} / Z_{C i} !\right)^{Z_{c i}}}{\prod_{i}\left(n_{A i} / Z_{C i} !\right)^{Z_{c i}}} \cdot \frac{\prod_{j}\left(n_{B j}^{*} / Z_{C j} !\right)^{Z_{c j}}}{\prod_{j}\left(n_{B j} / Z_{C j} !\right)^{Z_{c j}}} .
$$

where $n_{A i}^{*}$ and $n_{B j}^{*}$ are the number of atoms corresponding to the random distribution on the lattice sites and are given by the expressions

$$
\begin{aligned}
& n_{A i}^{*}=Z_{C i} \cdot n_{A}^{(Z-i+1)} n_{B}^{i} /\left(n_{A}+n_{B}\right)^{Z}, \\
& n_{B j}^{*}=Z_{C j} \cdot n_{A}^{j} \cdot n_{B}^{(Z-j+1)} /\left(n_{A}+n_{B}\right)^{Z} .
\end{aligned}
$$

$Z_{C i}$ is the number of configurations of $A$ atoms having $i B$ atoms around it and is given by

$$
Z_{C i}=\frac{Z !}{i !(Z-i) !} \text {. }
$$

As in quasi-chemical theory, assuming that the summation in eq. (3) can be replaced by the maximum term in the series, one gets two sets of expressions namely,

$$
\frac{\partial \ln \Omega}{\partial n_{A i}}=0 \text { for } 0 \leq i \leq Z \text {, }
$$

and

$$
\frac{\partial \ln \Omega}{\partial n_{B j}}=0 \text { for } 0 \leq j \leq Z \text {. }
$$

Substitution of eq. (5) in eq. (3) and of the resulting expression (without summation) in eqs. (9) and (10) and the application of mass balance constraints on the resulting set of expressions by a Lagrangian multiplier method leads to the expression

$$
\begin{aligned}
& n_{A i}={ }^{z} C i \alpha_{A}[\exp (i \lambda)]\left[\exp \left(-\frac{W_{0 A i}-k T \ln v_{A i}}{k T}\right)\right], \\
& n_{B j}={ }^{z} C j \alpha_{B}[\exp (-j \lambda)]\left[\exp \left(-\frac{W_{0 B j}-k T \ln v_{B j}}{k T}\right)\right],
\end{aligned}
$$

where $\ln \alpha_{A}, \ln \alpha_{B}$ and $\lambda$ are the multipliers and can be calculated from the following expressions which make use of mass and bond balance constraints.

$$
\begin{aligned}
& \alpha_{A}=n_{A}\left[\sum^{\mathrm{z}} C i\{\exp (i \lambda)\}\left\{\exp \left(-\frac{W_{0 A i}-k T \ln v_{A i}}{k T}\right)\right\}\right]^{-1}, \\
& \alpha_{B}=n_{B}\left[\sum^{{ }^{z}} C j\{\exp (-j \lambda)\}\left\{\exp \left(-\frac{W_{0 B j}-k T \ln v_{B j}}{k T}\right)\right\}\right]^{-1},
\end{aligned}
$$


and

$$
\begin{aligned}
\alpha_{A}\left[\sum i^{z} C i\{\exp (i \lambda)\}\left\{\exp \left(-\frac{W_{0 A i}-k T \ln v_{A i}}{k T}\right)\right\}\right] \\
=\alpha_{B}\left[\sum j^{z} C j\{\exp (-j \lambda)\}\left\{\exp \left(-\frac{W_{0 B j}-k T \ln v_{B j}}{k T}\right)\right\}\right] .
\end{aligned}
$$

From eqs. (14) and (15) one can derive the expression

$$
\begin{aligned}
& \frac{\left(n_{A i} / Z_{C i}\right)\left(n_{B j} / Z_{C j}\right)}{\left\{n_{A(i+1)} / Z_{C(i+1)}\right\}\left\{n_{B(j+1)} / Z_{C(j+1)}\right\}}=\frac{v_{A i} \cdot v_{B j}}{v_{A(i+1)} \cdot v_{B(j+1)}} \\
& \times\left[\exp \left(-\frac{W_{0 A i}-W_{0 A(i+1)}}{k T}\right)\right] \cdot\left[\exp \left(-\frac{W_{0 B j}-W_{0 B(j+1)}}{k T}\right)\right] .
\end{aligned}
$$

The above expression, which predicts an equilibrium insolution among atoms of different configurations, forms the quasi-chemical equivalent of the present approach.

It is clear from eqs. (11) (15) that to calculate the free energy of mixing one requires a knowledge of both the energies and free volumes of atoms as functions of their nearest neighbours. A number of such relationships have been suggested to predict the properties of non-electrolytes ${ }^{(8)}$. Most of these relationships are linear functions of the number of atoms of different types in the first coordination shell of atoms. These relationships have also been assumed to hold good in case of metallic solutions and in some cases give reasonably good results. But all these relationships are just empirical and fail in most of the cases. Mathieu et al. ${ }^{(9)(10)}$ have suggested empirically a parabolic type of relationship to explain the properties of binary and ternary solutions, but this also leads to approximate results particularly for entropies of mixing. Further, no explanation was given by the authors $^{(9)(10)}$ for the selection of such a relationship. For the calculations of free volumes a number of relationship for nonelectrolytes have also been suggested but these cannot be applied in the same form for metallic solution as in this case the process of mixing may involve a transfer of electrons from one component to the other. Using Hartree's method, Christman and Huntington ${ }^{(11)}$ have shown that there is a transfer of 0.4 electrons from potassium to sodium in equimolar liquid binary sodium-potassium solution. Oriani $^{(15)}$ has also stated that the interactions in metallic solutions are predominantly electronic interactions. In what follows, it will be attempted to arrive at suitable functions for the energy and free volumes of atoms in terms of their nearest neighbours from some semi-empirical considerations characterising metallic solutions.

In a solution of components $A$ and $B$, consider two clusters one of an $A$ atom surrounded by $Z A$ atoms and another of a $B$ atom surrounded by only $B$ atoms. Now remove an $A$ atom from the surroundings of $A$ and replace it by a $B$ atom and similarly a $B$ atom replaced by $A$ in the surroundings of $B$. The energies of both central $A$ and $B$ atoms will change. This change will be due to, (i) the difference in the electronegativities of the components and will cause a change in the ionic character of the bond between $A$ and $B$, (ii) change in potential field in which conduction band or valency electrons of $A$ and $B$ will move thus giving rise to change in density of states and wave functions and the energy of electrons. This part of energy can be solved only by perturbation theory of quantum mechanics and is further split into coulombic, exchange and correlation energies, (iii) change in Van der Waal's forces and (iv) change in free volume because of difference in sizes of atoms of components $A$ and $B$. A quantitative analysis of these energies requires a quantum mechanical formulation of this many particle problem and is extremely complicated and has till today not been solved for a general case, though in some cases e.g., for the system sodium-potassium approximate solutions ${ }^{(11)}$ are available. In the present case 
let us assume that this change in the energy of $A$ atom is equal to $\Delta W_{A 1}$ and is given by

$$
\Delta W_{A 1}=W_{0 A 1}-W_{0 A 0} \text {. }
$$

Now replace another $A$ atom from the surroundings of the $A$ atom by a $B$ atom. Let the change in the energies of $A$ atom be $\Delta W_{A 2}$ thus

$$
\Delta W_{A 2}=W_{0 A 2}-W_{0 A 1} .
$$

The effect of second $B$ atom on the energy of $A$ atom will be different from that of the first atom as among others the electron exchange capacity of $A$ atom after interaction with first $B$ atom shall be different. Thus, in general,

$$
\Delta W_{A Z} \neq \Delta W_{A 1} .
$$

Though the above statement is in contradiction with the basic assumption of quasichemical theory which assumes the energy of an atom to be equal to the sum of energies of bonds it makes with its nearest neighbours ${ }^{(7)}$ with each type of bond making the same contribution, yet it is better justifiable qualitatively in case of metallic systems.

In accordance with the above argument one can write the following inequality for the effect of replacement of further $A$ atoms by $B$ atoms around $A$

$$
\Delta W_{A 1} \neq \Delta W_{A 2} \neq \cdots \neq \Delta W_{A Z} .
$$

In order to arrive at the mathematical expressions for the energies of $\boldsymbol{A}$ atoms, let us now make the simplest empirical assumption that

$$
\Delta W_{A(i+1)}-\Delta W_{A i}=\delta_{A} \quad(a \text { constant }) .
$$

Similar assumption has been made by Wagner ${ }^{(12)}$ to explain the thermodynamic behaviour of a dilute interstitial component in a binary substitutional solution. On the basis of this assumption one can arrive at the following expression for $W_{0 A i}$

$$
W_{0 A i}=W_{0 A 0}+i \Delta W_{A 1}+i(i-1) \delta_{A} / 2 .
$$

Similarly one can write the following expression:

$$
W_{0 B j}=W_{0 B 0}+j \Delta W_{B 1}+j(j-1) \delta_{B} / 2 .
$$

It may be pointed out that for $\delta_{A}$ and $\delta_{B}$ equal to zero, one gets the same expression for the energies of $A$ and $B$ atoms as are assumed in quasi-chemical theory. It will be assumed further on the basis of already put forward arguments that natural logarithms of free volumes also obeys the expressions of the type represented by eqs. (22) and (23) i.e.,

$$
\ln v_{A i}=\ln v_{A 0}+i \Delta \ln v_{A 1}+i(i-1) \eta_{A} / 2,
$$

and

$$
\ln v_{B j}=\ln v_{B 0}+j \Delta \ln v_{B j}+j(j-1) \eta_{B} / 2 .
$$

Once the forms of energy and free volume function are derived let us substitute them in eq. (15) to evaluate the lagrangian multiplier. Thus

$$
\begin{aligned}
n_{A}\left[\sum i^{z} C i\{\exp (i \lambda)\}\left\{\exp \left(-\frac{i\left(\Delta W_{A 1}-R T \Delta \ln v_{A 1}\right)}{k T}\right)\right\}\left\{\exp \left(-\frac{\left(\delta_{A}-k T \eta_{A}\right) i(i-1)}{2 k T}\right)\right\}\right] \\
\times\left[{ }^{z} C i\{\exp (i \lambda)\}\left\{\exp \left(-\frac{i\left(\Delta W_{A i}-k T \ln v_{A i}\right.}{k T}\right)\right\}\left\{\exp \left(-\frac{i(i-1)\left(\delta_{A}-k T \eta_{A}\right)}{k T}\right)\right\}\right]^{-1} \\
=n_{B}\left[\sum j^{z} C j\{\exp (-j \lambda)\}\left\{\exp \left(-\frac{j\left(\Delta W_{B 1}-k T \Delta \ln v_{B 1}\right)}{k T}\right)\right\}\left\{\exp \left(-\frac{\left(\delta_{B}-k T \eta_{B}\right) j(j-1)}{2 k T}\right)\right\}\right] \\
\left.\times\left[\sum^{z} C j \exp (-j \lambda)\left\{-\frac{j\left(\Delta W_{B 1}-k T \Delta \ln v_{B 1}\right)}{k T}\right)\right\}\left\{\exp \left(-\frac{\left(\delta_{B}-k T \eta_{B}\right) j(j-1)}{k T}\right)\right\}\right]^{-1} \cdot
\end{aligned}
$$

Equations (4), (11), (12) and (26) which describe the thermodynamic functions of a system are complex and require iterative solutions with the help of a computer. To obtain explicit solutions for discussion and practical purposes, let us consider two simplified cases. First let us consider that the volume terms and the energy terms divided by $k T$ in eqs. (26), (11) (15) are so small that the exponential terms containing them can be set equal to unity. On this assumption, one 
obtains the following expression from eqs. (26):

$$
\exp \lambda=\frac{n_{B}}{n_{A}}
$$

Hence from eqs. (11) and (12) one gets

$$
n_{A i}={ }^{z} C i \cdot\left(N_{A}\right)^{Z-i} \cdot\left(N_{B}\right)^{i} \cdot n_{A}=n_{A i}^{*}
$$

and

$$
n_{B j}={ }^{Z} C j\left(N_{A}\right)^{j} \cdot\left(N_{B}\right)^{Z-j} \cdot n_{B}=n_{B i j}^{*},
$$

respectively. This assumption corresponds to Hildebrand's regular solution theory ${ }^{(1)}$ and also to Zeroth or Bragg-Williams approximation in statistical theory ${ }^{(8)}$ of cooperative phenomenon.

Substitution of eqs. (28) and (29) in eq. (5) and of the resulting expression in eq. (3) leads, with the help of eq. (4), to the following expression for the excess free energy of mixing $\left(\Delta G_{\text {mix }}^{\text {ex }}\right)$ for the system:

$$
\begin{aligned}
\Delta G_{\mathrm{mix}}^{\mathrm{ex}}= & Z N_{A} N_{B} N\left[\left(\Delta W_{A 1}+\Delta W_{B 1}\right)+\delta_{B}(Z-1) / 2+(Z-1)\left(\delta_{A}-\delta_{B}\right) N_{B} / 2\right] \\
& -Z R T N_{A} N_{B}\left[\Delta \ln v_{A 1}+\Delta \ln v_{B 1}+\eta_{B}(Z-1) / 2+(Z-1)\left(\eta_{A}-\eta_{B}\right) N_{B} / 2\right] .
\end{aligned}
$$

Relevant differentiation of the above expression yields the following expressions for the enthalpy $\left(\Delta H_{\mathrm{mix}}\right)$ and excess entropy $\left(\Delta S_{\mathrm{mix}}^{\mathrm{ex}}\right)$ of mixing

$$
\Delta H_{\text {mix }}=Z N_{A} N_{B} N\left[\Delta W_{A 1}+\Delta W_{B 1}+(Z-1) \delta_{B} / 2+(Z-1)\left(\delta_{A}-\delta_{B}\right) N_{B} / 2\right],
$$

and

$$
\Delta S_{\mathrm{mix}}^{\mathrm{ex}}=Z R N_{A} N_{B}\left[\Delta \ln v_{A 1}+\Delta \ln v_{B 1}+\eta_{B}(Z-1) / 2+(Z-1)\left(\eta_{A}-\eta_{B}\right) N_{B} / 2\right] .
$$

Equations (30) (32) predict a linear relationship between the above-mentioned excess thermodynamic properties divided by the products of mole fractions of the components and the mole fraction of either of the components.

As a second approximation of eqs. (11) (15) and eq. (26), let us assume that the parabolic terms in these expressions are so small that their exponentials can be set equal to one. This assumption corresponds to the first approximation or Bethe's approximation in the statistical theory of cooperative phenomenon ${ }^{(8)}$ or strictly regular solution model ${ }^{(2)}$. Using this assumption, one can simplify eqs. (13), (14) and (26). Making use of these simplified expressions and taking series expansions of the square root term in $\exp (\lambda)$ expression and natural logarithmic terms in $\Delta G_{\text {mix }}^{\text {ex }}$, one can arrive at the following expressions for excess properties of mixing:

$$
\begin{aligned}
\Delta G_{\mathrm{mix}}^{\mathrm{ex}}= & Z N_{A} N_{B}\left[N\left(\Delta g_{A 1}+\Delta g_{B 1}\right)+(Z-1) N\left(\delta_{A}-k T \eta_{A}\right) N_{B} / 2+(Z-1) N\left(\delta_{B}-k T \eta_{B}\right) N_{A} / 2\right] \\
& \left.-\frac{\left[N\left(\Delta g_{A 1}+\Delta g_{B 1}\right)\right]^{2}}{R T} \cdot Z N_{A}^{2} N_{B}^{2}+Z(Z-1)\left[\delta_{B}-k T \eta_{B}\right) N_{B}+\left(\delta_{A}-k T \eta_{A}\right) N_{A}\right] \\
& \times \frac{N\left(\Delta g_{A 1}+\Delta g_{B 1}\right)}{R T} \cdot N_{A}^{2} N_{B}^{2} \\
\Delta H_{\text {mix }}= & Z\left[N\left(\Delta W_{A 1}+\Delta W_{B 1}\right)+(Z-1)\left(\delta_{A} N_{B}+\delta_{B} N_{A}\right) / 2\right] \cdot N_{A} N_{B} \\
& +\left(\Delta W_{A 1}+\Delta W_{B 1}\right) \cdot \frac{N\left(\Delta g_{A 1}+\Delta g_{B 1}\right)}{R T} \cdot Z N_{A}^{2} N_{B}^{2}+(Z-1) Z\left(\delta_{B} N_{B}+\delta_{A} N_{A}\right) \\
& \times \frac{\Delta g_{A 1}+\Delta g_{B 1}}{R T} \cdot N^{2} N_{A}^{2} N_{B}^{2}+Z(Z-1)\left[\left(\delta_{B}-k T \eta_{B}\right) N_{B}+\left(\delta_{A}-k T \eta_{A}\right) N_{A}\right] \\
& \times \frac{\Delta W_{A 1}+\Delta W_{B 1}}{R T} \cdot N^{2} N_{A}^{2} N_{B}^{2}
\end{aligned}
$$




$$
\begin{aligned}
\Delta S_{\mathrm{mix}}^{\mathrm{ex}}= & Z R\left[\Delta\left(\ln v_{A 1}\right)+\Delta\left(\ln v_{B 1}\right)+(Z-1)\left(\eta_{A} N_{B}+\eta_{B} N_{A}\right) / 2\right] N_{A} N_{B} \\
& -Z R\left[\frac{\left(\Delta g_{A 1}+\Delta g_{B 1}\right) N}{R T}\right]^{2} N_{A}^{2} N_{B}^{2}+Z(Z-1)\left(\eta_{B} N_{A}+\eta_{A} N_{B}\right) \cdot \frac{\Delta g_{A 1}+\Delta g_{B 1}}{R T} \cdot N R N_{A}^{2} N_{B}^{2} \\
& -Z(Z-1)\left[\left(\delta_{A}-k T \eta_{A}\right) N_{A}+\left(\delta_{B}-k T \eta_{B}\right) N_{B}\right] \cdot \frac{\Delta W_{A 1}+\Delta W_{B 1}}{R T^{2}} \cdot N^{2} N_{A}^{2} N_{B}^{2}
\end{aligned}
$$

where

$$
\Delta g_{A 1}=\Delta W_{A 1}-k T \Delta \ln v_{A 1},
$$

and

$$
\Delta g_{B 1}=\Delta W_{B 1}-k T \Delta \ln v_{B 1} .
$$

Comparison of eqs. (30) (32) with eqs. (33) (35) leads to the following facts: (i) Equations (33) and (34) can be separated into two parts, namely, a part corresponding to random mixing (which forms the first term in these expressions) and another part corresponding to the preferential distribution of atoms in solution (which consists of the rest of the terms in these expressions). The preferential distribution part depends upon the difference in the energies between like and unlike atoms. It is evident from eqs. (30), (31), (33) and (34) that if $\Delta G_{\mathrm{mix}} / N_{A} N_{B}$ and $\Delta H_{\text {mix }} / N_{A} N_{B}$ are plotted against the mole fraction of the components, the line joining the two ends of the curve forms the contribution of the random mixing part whereas the deviation from this line forms the contribution due to preferential distribution. It can be further concluded from eq. (33) that if the energies of interaction between unlike atoms are more negative than the like ones, the preferential distribution part is also negative showing thereby that the solution will tend to the state in which more of bonds between unlike atoms are formed than in the case of random mixing. (ii) Comparision of eq. (35) with eq. (32) shows that the excess entropy of mixing in eq. (35) consists of two parts, namely, a non-configurational part which is due to the changes in free volume on mixing and a configurational part arising from the preferential distribution of atoms due to the difference in interaction energies between like and unlike atoms in solution. The configurational part is equal to zero in the case of randomly distributed solutions and is always negative in the case of preferentially distributed solutions as is clear from eqs. (32) and (35) respectively. (iii) The preferential configurational effects are seen to be inverse functions of temperature from eqs. (33) (35).

\section{Application and Discussion}

To evaluate the thermodynamic properties of a binary substitutional system from either eqs. (30) $\sim(32)$ or eqs. (33) $\sim(35)$ one requires a knowledge of eight parameters, namely, $\Delta W_{A 1}, \Delta W_{B 1}, \Delta \ln v_{A 1}, \Delta \ln v_{B 1}, \delta_{A}, \delta_{B}, \eta_{A}$ and $\eta_{B}$. No data is available as yet on these parameters so these equations can be considered as ones describing the form of thermodynamic functions. To make use of these equations, one requires expressing these theoretical parameters in the terms of some experimentally determinable variables. These eight experimentally determinable parameters can be partial molar heats $\left(\Delta \bar{H}_{A \infty}, \Delta \bar{H}_{B \infty}\right)$ and entropies $\left(\Delta \bar{S}_{A \infty}^{\text {ex }}, \Delta \bar{S}_{B \infty}^{\text {ex }}\right)$ at infinite dilution of the components and their heats $\left(\varepsilon_{A}^{A}, \varepsilon_{B}^{B}\right)$ and entropies $\left(\sigma_{A}^{A}, \sigma_{B}^{B}\right)$ of interaction. Reformulation of eqs. (30) $\sim(32)$ in terms of these variables leads to the expressions

$$
\begin{aligned}
& \Delta G_{\mathrm{mix}}^{\mathrm{ex}}=\left(\Delta \bar{G}_{A \infty} N_{B}+\Delta \bar{G}_{B \infty} N_{A}\right) N_{A} N_{B}, \\
& \Delta H_{\mathrm{mix}}=\left(\Delta \bar{H}_{A \infty} N_{B}+\Delta \bar{H}_{B \infty} N_{A}\right) N_{A} N_{B}, \\
& \Delta S_{\mathrm{mix}}^{\mathrm{ex}}=\left(\Delta \bar{S}_{A \infty} N_{B}+\Delta \bar{S}_{B \infty} N_{A}\right) N_{A} N_{B} .
\end{aligned}
$$

In Fig. 1, $\Delta G^{\text {ex }} / N_{A} N_{B}$ is plotted against composition for the systems $\mathrm{Ag}-\mathrm{X}$ (where $\mathrm{X}$ represents the other substitutional component). There is no particular reason for the selection of these systems except that these have been 


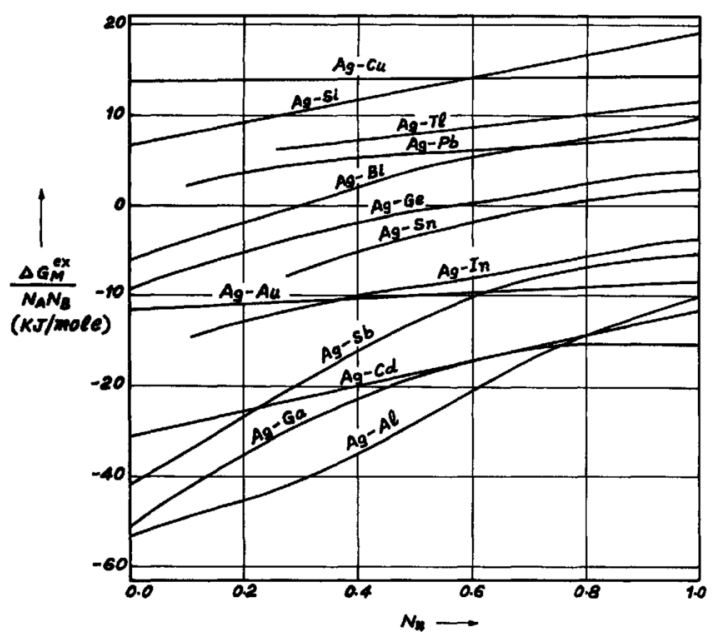

Fig. 1 Excess free energy of mixing of silver base binary molten metallic substitutional solutions.

extensively studied and that their thermodynamic data is known to a good degree of accuracy ${ }^{(13)}$. It may be mentioned here that those components which differ in their atomic sizes by about 15\% (according to HumeRothery Rules) are considered to form substitutional solutions.

Figure 1 shows that eq. (30) is followed by some of binary systems e.g. $\mathrm{Ag}-\mathrm{Si}, \mathrm{Ag}-\mathrm{Cu}$, $\mathrm{Ag}-\mathrm{Ti}$ and $\mathrm{Ag}-\mathrm{Au}$ whereas others show a deviation from linear behaviour indicating thereby that the atoms in the system are not randomly distributed. To quantitatively explain the behaviour of these systems, one has to use eq. (33) which on substitution of experimentally determinable parameters yields the expression

$$
\begin{aligned}
\Delta G_{\mathrm{mix}}^{\mathrm{ex}}= & \left(\Delta \bar{G}_{B \infty}^{\mathrm{ex}} N_{A}+\Delta \bar{G}_{A \infty \infty}^{\mathrm{ex}} N_{B}\right) N_{A} N_{B} \\
& +\left[\left(\varepsilon_{A}^{A} / 2-2 \Delta \bar{G}_{B \infty}^{\mathrm{ex}}+\Delta \bar{G}_{A \infty}^{\mathrm{ex}}\right) N_{B}\right. \\
& \left.+\left(\varepsilon_{B}^{B} / 2-2 \Delta \bar{G}_{A \infty}^{\mathrm{ex}}+\Delta \bar{G}_{B \infty}^{\mathrm{ex}}\right) N_{A}\right] N_{A}^{2} N_{B}^{2} .
\end{aligned}
$$

As the values of $\Delta \bar{G}_{A \infty \infty}^{\mathrm{ex}}, \Delta \bar{G}_{B \infty 0}^{\mathrm{ex}}, \varepsilon_{A}^{A}, \varepsilon_{B}^{B}$ are not available, these are determined from available data on partial molar excess free energies by plotting them against composition for the binary system in question. These parameters are given in Table 1 for the systems plotted in Fig. 1. Calculations, with the help of this data and eq. (41), have shown that in almost all the cases in Fig. 1, the maximum deviation of the calculated values from the experimental ones was less than 3\% which is far less than that of other approaches and lies within the experimental range of accuracy of determined data. For the sake of comparison of predicted values by various models with the experimentally determined one, a typical system shall be considered later. Let us now come to the calculation of heat and entropy of mixing. With the help of eqs. (34) and (35) and the definitions of experimentally determinable parameters $\rho_{A}^{A}, \rho_{B}^{B}, \sigma_{A}^{A}$ and $\sigma_{B}^{B}$ one can arrive at the following expressions for excess integral molar heats and entropies of mixing.

\begin{tabular}{|c|c|c|c|c|c|}
\hline System & $\begin{array}{l}\text { Temperature } \\
(\mathbf{K})\end{array}$ & $\bar{G}_{A}^{\operatorname{ex}}(\mathrm{J})$ & $\bar{G}_{B}^{\mathrm{ex}}(\mathrm{J})$ & $T R \varepsilon_{A}^{A}(\mathrm{~J})$ & $R T \varepsilon_{B}^{B}(\mathrm{~J})$ \\
\hline $\mathrm{Ag}-\mathrm{Si}$ & 1700 & 19110 & 6510 & -63420 & 12180 \\
\hline $\mathrm{Ag}-\mathrm{Cu}$ & 1423 & 13440 & 13440 & -26880 & -26880 \\
\hline $\mathrm{Ag}-\mathrm{Ti}$ & 975 & 11340 & 6510 & -32340 & -3360 \\
\hline $\mathrm{Ag}-\mathrm{Bi}$ & 1000 & 9660 & -6300 & -39900 & 54600 \\
\hline $\mathrm{Ag}-\mathrm{Pb}$ & 1273 & 7560 & 1680 & -18900 & 28140 \\
\hline $\mathrm{Ag}-\mathrm{Ge}$ & 1250 & 3780 & -9660 & -15960 & 63420 \\
\hline $\mathrm{Ag}-\mathrm{Sn}$ & 1250 & 1680 & -7980 & -11760 & 49560 \\
\hline $\mathrm{Ag}-\mathrm{In}$ & 1100 & -3780 & -14700 & -11340 & 62160 \\
\hline $\mathrm{Ag}-\mathrm{Sb}$ & 1250 & -5460 & -31500 & 2100 & 140700 \\
\hline $\mathrm{Ag}-\mathrm{Au}$ & 1350 & -8610 & -9660 & 15120 & 21420 \\
\hline $\mathrm{Ag}-\mathrm{Al}$ & 1273 & -10080 & -36960 & -25200 & 113820 \\
\hline $\mathrm{Ag}-\mathrm{Cd}$ & 1223 & -11760 & -25830 & -4620 & 79800 \\
\hline $\mathrm{Ag}-\mathrm{Ga}$ & 1000 & -15540 & -26880 & 31080 & 102480 \\
\hline
\end{tabular}

Table 1 Excess partial molar free energy at infinite dilution and interaction free energy for binary molten metallic substitutional solutions. 


$$
\begin{aligned}
\Delta H_{\mathrm{mix}}= & \left(\Delta \bar{H}_{A \infty} N_{B}+\Delta \bar{H}_{B \infty} N_{A}\right) N_{A} N_{B} \\
& +\left[\left(\rho_{A}^{A} / 2-2 \Delta \bar{H}_{B \infty}+\Delta \bar{H}_{A \infty}\right) N_{B}\right. \\
& \left.+\left(\varepsilon_{B}^{B} / 2-2 \Delta \bar{H}_{A \infty}+\Delta \bar{H}_{B \infty}\right) N_{A}\right] N_{A}^{2} N_{B}^{2},
\end{aligned}
$$

and

$$
\begin{aligned}
\Delta S_{\mathrm{mix}}^{\mathrm{ex}}= & \left(\Delta \bar{S}_{A \infty}^{\mathrm{ex}} N_{B}+\Delta \bar{S}_{B \infty}^{\mathrm{ex}} N_{A}\right) N_{A} N_{B} \\
& +\left[\left(\sigma_{A}^{A} / 2-2 \Delta \bar{S}_{B \infty}^{\mathrm{ex}}+\Delta \bar{S}_{A \infty}^{\mathrm{ex}}\right) N_{B}\right. \\
& \left.+\left(\sigma_{B}^{B} / 2-2 \Delta \bar{S}_{A \infty}^{\mathrm{ex}}+\Delta \bar{S}_{B \infty}^{\mathrm{ex}}\right) N_{A}\right] N_{A}^{2} N_{B}^{2}
\end{aligned}
$$

Applicability of the above expressions is also tested on silver-base systems. The data for enthalpy and excess entropy of mixing is plotted in Figs. 2 and 3 respectively. The calculated parameters in a similar way to the case of free energy of mixing are given in Table 2. These calculated values with the help of eqs. (42) and (43) agree well with experimental ones.

In order to compare the present approach with the others, the calculated and experimental values of excess integral molar free energy, heat and entropy of mixing are plotted for the system Ag-Ge in Fig. 4. These two metals, which will form a substitutional solution as their atomic sizes differ by less than $15 \%$, have vastly different crystal structure and valency which, as already stated, will affect

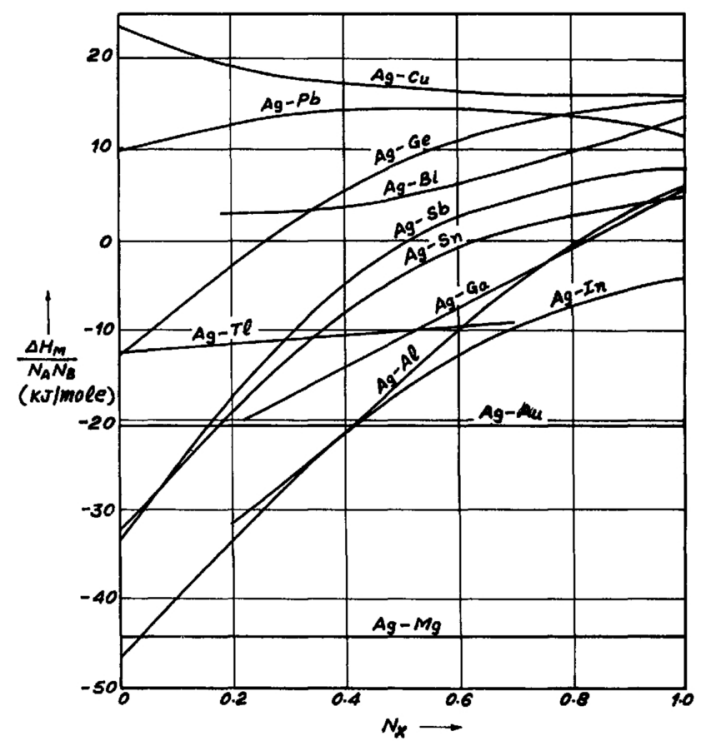

Fig. 2 Heat of mixing of silver base binary molten metallic substitutional solutions.

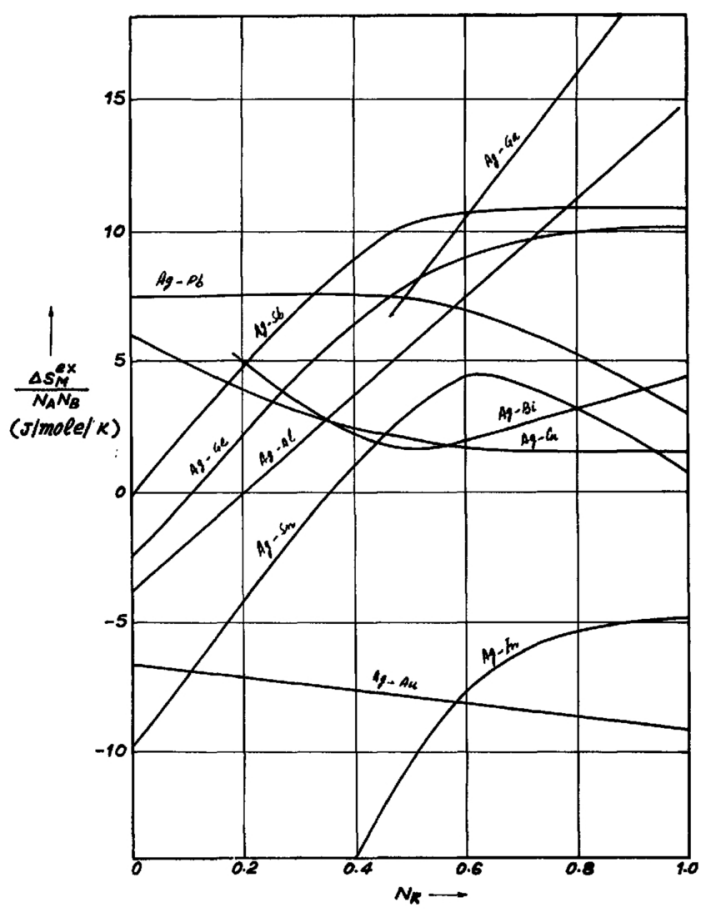

Fig. 3 Excess entropy of mixing of silver base binary molten metallic substitutional solutions.

their interaction energies. Figure 4 shows that the present approach is capable of explaining the thermodynamic data of substitutional solutions of such vastly different metals to a good degree of accuracy. It also shows that the regular solution model and quasi-chemical theory which lead to symmetrical calculated values with respect to composition, do not show an agreement with the experimental value. The same remark can be made regarding these approaches for the explanation of thermodynamic functions of other assymmetrical systems. Only the model which explains to some extent the assymmetrical behaviour is subregular solution model ${ }^{(14)}$. But because of the random mixing assumption and not taking into consideration the non-configuration changes in entropy, it is unable to explain the excess entropy and enthalpy functions and leads only to linear relationships which in many cases do not hold good. Another model, namely a surrounded atom model, which is capable of explaining assymmetrical behaviour is based on an empirical parabolic assumption of atomic energies and requires computed 
Table 2 Partial molar heat and entropies of mixing at infinite dilution and heat of interaction for silver base binary molten metallic substitutional solutions.

\begin{tabular}{|c|c|c|c|c|c|c|c|c|}
\hline System & $\underset{(J)}{\Delta \bar{H}_{A \infty}}$ & $\underset{(\mathbf{J})}{\Delta \bar{H}_{B \infty}}$ & $\begin{array}{l}\rho_{A}^{A} \\
(J)\end{array}$ & $\begin{array}{l}\rho_{B}^{B} \\
(\mathrm{~J})\end{array}$ & $\frac{\Delta \overline{\boldsymbol{S}}_{A \infty}^{\text {ex }}}{(\mathbf{J} / \mathbf{K})}$ & $\underset{(\mathbf{J} / \mathbf{K})}{\Delta \bar{S}_{\mathbf{B}_{\infty}}^{\mathbf{x}}}$ & $\begin{array}{c}\sigma_{A}^{A} \\
(\mathbf{J} / \mathbf{K})\end{array}$ & $\begin{array}{c}\sigma_{B}^{B} \\
(\mathbf{J} / \mathbf{K})\end{array}$ \\
\hline $\mathrm{Ag}-\mathrm{Cu}$ & 16170 & 23730 & -79800 & -81480 & 1470 & 6020 & -2940 & -35840 \\
\hline $\mathrm{Ag}-\mathrm{Ge}$ & 15960 & -13020 & -46200 & 126000 & 10080 & -2520 & -20860 & 52089 \\
\hline $\mathrm{Ag}-\mathrm{Bi}$ & 13860 & 3150 & -72240 & -5040 & 4340 & 5110 & -21700 & -38220 \\
\hline $\mathrm{Ag}-\mathrm{Sb}$ & 8400 & -33600 & -33600 & 252000 & 10780 & -140 & -21140 & 49000 \\
\hline $\mathrm{Ag}-\mathrm{Ga}$ & 58880 & -20580 & -41160 & 70560 & 18200 & 6860 & -59080 & 8960 \\
\hline $\mathrm{Ag}-\mathrm{Sn}$ & -5040 & -32340 & -31920 & 196560 & 700 & -9800 & -32060 & 74900 \\
\hline $\mathrm{Ag}-\mathrm{Ti}$ & -9030 & -12390 & 11340 & 31500 & - & - & - & - \\
\hline $\mathrm{Ag}-\mathrm{In}$ & -3780 & -35700 & -17220 & 158760 & -4830 & -14000 & 5740 & 74200 \\
\hline $\mathrm{Ag}-\mathrm{Au}$ & -20580 & -20580 & 41160 & 41160 & -9100 & -6580 & 23240 & 8120 \\
\hline $\mathrm{Ag}-\mathrm{Al}$ & - & - & - & - & 14840 & -3780 & 66920 & 44800 \\
\hline $\mathrm{Ag}-\mathrm{Pb}$ & - & - & - & - & 2940 & 7420 & 22820 & -14840 \\
\hline
\end{tabular}

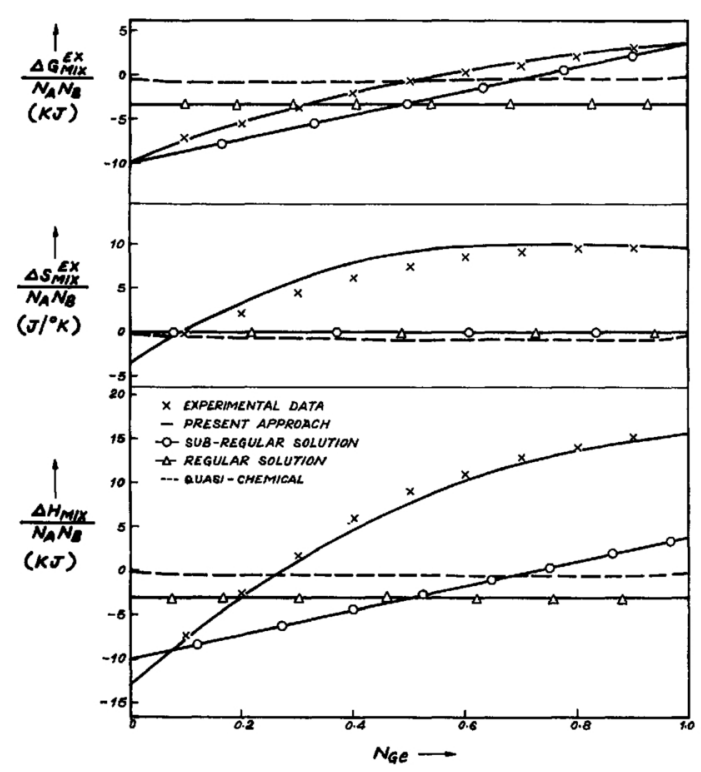

Fig. 4 Comparison of various approaches.

solutions. But this model, also, as shown in Ref. (9), does not explain the excess entropy of mixing. Along with other approaches the present model also suffers from the drawback that the parameters required for calculating thermodynamic properties are determined from the knowledge of these properties at some composition which is that corresponding to infinitely dilute solutions in the present case. Thus this model, as also the others, can only be used for calculating the thermodynamic values at a desired composition from those at the other. This drawback can be overcome by a quantum mechanical analysis or by finding empirical relationships between thermodynamic parameters and the properties of metals. The author is of the view that the second method i.e. empirical analysis of the data will be more appropriate. For this purpose $\left(\Delta g_{A 1}+\Delta g_{B 1}\right)$, $\left(\delta_{A}-k T \eta_{A}\right)$ and $\left(\delta_{B}-k T \eta_{B}\right)$ are calculated from $\Delta \bar{G}_{A \infty 0}^{\mathrm{ex}}, \Delta \bar{G}_{B \infty 0}^{\mathrm{ex}}, \varepsilon_{A}^{A}$ and $\varepsilon_{B}^{B}$ and are given in Table 3 for the systems plotted in Fig. 1. Data on systems $\mathrm{Ag}-\mathrm{Cu}$ and $\mathrm{Ag}-\mathrm{Au}$ show that the parameters for systems belonging to same groups have low values as in such cases the components do not differ largely in their crystal structure, valency, electronegativities and atomic radii. A more negative $\left(\Delta g_{A 1}+\Delta g_{B 1}\right)$ value for $\mathrm{Cu}-\mathrm{Ag}$ than $\mathrm{Ag}-\mathrm{Au}$ may be due to effect of conduction band electrons. Analysis of systems from the same period (i.e. $\mathrm{Ag}-\mathrm{In}$, $-\mathrm{Sn},-\mathrm{Sb}$ ) shows that free energy and enthalpy values become more negative with increase in electronegativity difference and the differences in structure and atomic size tend to lead to positive deviations. The entropy parameters seem to be affected mainly by the size difference. Thus it is seen that the factors affecting the parameters shall be the electronegativity, valency, atomic size, crystal structure and interaction with conduction band. For a quantitative empirical analysis more data is required.

\section{Acknowledgements}

The author thankfully acknowledges the help rendered by Sri Vinod Kumar for prepara- 
Table 3 Interaction functions for binary silver base molten metallic substitutional solutions.

\begin{tabular}{|c|c|c|c|c|c|c|c|c|c|}
\hline Systems & $\begin{array}{c}\Delta g_{A 1}+ \\
\Delta g_{B 1} \\
(\mathbf{J})\end{array}$ & $\begin{array}{l}\delta_{A}- \\
k T \eta_{A} \\
(\mathrm{~J})\end{array}$ & $\begin{array}{l}\delta_{B}- \\
\left(J \eta_{B}\right.\end{array}$ & $\begin{array}{c}\Delta W_{A 1}+ \\
\Delta W_{B 1} \\
(\mathrm{~J})\end{array}$ & $\begin{array}{l}\eta_{A} \\
(J)\end{array}$ & $\begin{array}{l}\eta_{B} \\
(\mathfrak{J})\end{array}$ & $\begin{array}{c}\Delta \ln v_{\boldsymbol{A 1}}+ \\
\Delta \ln v_{B 1} \\
(\mathbf{J} / \mathbf{K})\end{array}$ & $\begin{array}{c}\eta_{A} \\
(\mathrm{~J} / \mathrm{K})\end{array}$ & $\begin{array}{c}\eta_{B} \\
(\mathrm{~J} / \mathbf{K})\end{array}$ \\
\hline $\mathrm{Ag}-\mathrm{Si}$ & 20 & 420 & 140 & - & - & - & - & - & - \\
\hline $\mathrm{Ag}-\mathrm{Cu}$ & -10 & 300 & 300 & -10920 & 2783 & 2955 & -0.925 & 0.21 & 0.225 \\
\hline $\mathrm{Ag}-\mathrm{Tl}$ & 20 & 250 & 140 & 2430 & -740 & -810 & 0.3 & -0.12 & -0.18 \\
\hline $\mathbf{A g}-\mathbf{B i}$ & -330 & 290 & -70 & 2360 & -220 & -450 & 0.324 & -0.06 & -0.46 \\
\hline $\mathrm{Ag}-\mathrm{Pb}$ & 8400 & -1700 & -1830 & -1550 & 610 & 570 & -0.94 & 0.22 & 0.23 \\
\hline $\mathrm{Ag}-\mathrm{Ge}$ & -500 & 190 & -100 & 26250 & -5480 & -6100 & 3.22 & -0.54 & -0.58 \\
\hline $\mathrm{Ag}-\mathrm{Sn}$ & 1300 & -250 & -470 & -13200 & 3290 & 2210 & -1.4 & 0.34 & 0.26 \\
\hline $\mathrm{Ag}-\mathrm{In}$ & 2230 & -580 & -820 & 4080 & 820 & -1680 & 0.2 & 0.15 & -0.09 \\
\hline $\mathrm{Ag}-\mathrm{Sb}$ & -3780 & 720 & 140 & 840 & - & -930 & 0.45 & -0.07 & -0.10 \\
\hline $\mathrm{Ag}-\mathrm{Au}$ & -15 & -190 & -210 & 0 & 460 & 460 & 0.001 & 0.06 & 0.06 \\
\hline $\mathrm{Ag}-\mathrm{Al}$ & -3700 & 590 & 30 & - & - & - & - & - & - \\
\hline $\mathrm{Ag}-\mathrm{Cd}$ & -10 & -260 & -570 & - & - & - & - & - & - \\
\hline $\mathrm{Ag}-\mathrm{Ga}$ & 1230 & -620 & -870 & -3550 & 920 & 330 & -0.58 & 0.19 & 0.14 \\
\hline
\end{tabular}

tion of this manuscript and Dr. E. Caune for providing the facilities for doing calculations.

\section{Nomenclature}

$g\left(n_{A}, n_{B}, n_{A i}, n_{B j}\right) \quad$ Number of different configurations corresponding to a given energy value

$\Delta g_{A 1}, \Delta g_{B 1} \quad$ Free energy change per atom when an atom in the surrounding of $A$ or $B$ is replaced by $B$ or $A$ respectively in pure $A$ or $B$

$\Delta G_{\text {mix }}, \Delta G_{\text {mix }}^{\text {ex }} \quad$ Free energy and excess free energy of mixing respectively per mole of solution $\Delta \bar{G}_{A \infty}^{\mathrm{ex}}, \Delta \bar{G}_{B \infty}^{\mathrm{ex}} \quad$ Partial molar excess free energies of components $A$ and $B$ respectively at infinite dilution

$\Delta H_{M} \quad$ Heat of mixing per mole of solution

$\Delta \bar{H}_{A \infty}, \Delta \bar{H}_{B \infty} \quad$ Partial molar heats of mixing of components $A$ and $B$ respectively

$k$

Boltzmann's constants

$n_{A}, n_{B}$

$n_{A i}^{*}, n_{A i}$

Number of atoms of components $A$ and $B$ respectively in solution

$n_{B j}^{*}, n_{B j}$

$N_{A}, N_{B}$

$R$

Number of atoms of $A$ surrounded by $i$ atoms of $B$ in a randomly distributed solution and solution under equilibrium respectively

Number of atom of $B$ surrounded by $j$ atoms of $A$ in a randomly distributed solution and solution under equilibrium respectively

Mole fraction of components $A$ and $B$ respectively

Universal gas constant

$\Delta S_{\mathrm{mix}}^{\mathrm{ex}}$

$\Delta \bar{S}_{A \infty}^{\mathrm{ex}}, \Delta \bar{S}_{B \infty}^{\mathrm{ex}}$

Excess entropy of mixing

Partial molar excess entropies of components $A$ and $B$ respectively at infinite dilution

$T$

$v_{A i}, v_{B j}$

$\Delta \ln v_{A i}, \Delta \ln v_{B j}$

$W_{0}$

$W_{O A i}, W_{O B j}$

$W_{A i}, W_{B j}$
Absolute temperature

Free volumes of atoms $A$ and $B$ respectively, surrounded by $i$ atoms of $B$ and $j$ atoms of $A$ respectively

Change in natural logarithms of free volumes of $A$ (surrounded initially only by $A$ ) and of $B$ (surrounded initially only by $B$ atoms) respectively on replacement of an $A$ atom by $B$ and a $B$ atom by $A$ in their surroundings respectively Energy of the system when all atoms occupy their mean positions

Energies of $A$ and $B$ atoms at their mean positions respectively when they are surrounded by $i$ atoms of $B$ and $j$ atoms of $A$ respectively

Energies of $A$ and $B$ atoms respectively when they are surrounded by $i$ atoms of $B$ and $j$ atoms of $A$ respectively 
$\Delta W_{A 1}, \Delta W_{B 1} \quad$ Change in energy of $A$ atom (initially surrounded only by $A$ atoms) and of $B$ atoms (initially surrounded only by $B$ atoms) respectively when one surrounding atom of $A$ is replaced by $B$ and one surrounding atom of $B$ is replaced by $A$ respectively

$Z \quad$ Coordination number

$v_{A}, v_{B}$

Activity coefficients of components $A$ and $B$ respectively

$\varepsilon_{A}^{A}, \varepsilon_{B}^{B}$

Free energies of self interactions of $A$ and $B$ respectively in their dilute solutions

$\rho_{A}^{A}, \rho_{B}^{B}$

$\sigma_{A}^{A}, \sigma_{B}^{B}$

Enthalpies of self interactions of $A$ and $B$ respectively in their dilute solutions

Entropies of self interactions of $A$ and $B$ respectively in their dilute solutions Configurational partition function of the system

\section{REFERENCES}

(1) J. H. Hildebrandt: J. Amer. Chem. Soc., 6 (1929), 221.

(2) E. A. Guggenheim: Mixtures, Oxford University Press, (1952).

(3) J. G. Kirkwood: J. Chem. Phys., 6 (1938), 70.

(4) E. Ising: Z. Physik, 31 (1925), 253.

(5) C. H. P. Lupis and J. F. Elliot: Acta Met., 15 (1967), 265.

(6) J. C. Mathieu et al.: J. Chem. Phys., 62 (1965), 1289.

(7) M. L. Kapoor: Int. Met. Reviews, 20 (1975), 150.

(8) A. Munster: Statistische Thermodynamik, Springer
Verlag, Berlin, (1956).

(9) J. C. Mathieu et al.: Advances in Physics, 16 (1967), 523

(10) J. C. Mathieu et al.: J. Chem. Phys., 66 (1969), 1238.

(11) J. R. Christman and H. B. Huntigton: Phys. Rev., 139 (1965), 483.

(12) C. Wagner: Acta Met., 21 (1973), 1297.

(13) H. Hultgren et al.: Selected Values of Thermodynamic Properties of Metals and Alloys, Wiley, N.Y., (1963).

(14) H. K. Hardy: Acta Met., 1 (1953), 202.

(15) R. A. Oriani: J. Chem. Phys., 19(1) (1951), 93. 